\title{
Immuntherpie beim Melanom: Nutzen trotz Behandlungsabbruch
}

\begin{abstract}
Selbst nach dem Abbrechen der Therapie scheinen viele Patienten mit fortgeschrittenem malignem Melanom Nutzen aus der Kombination von Nivolumab und Ipilimumab zu ziehen. Das ergab eine retrospektive Analyse der Daten aus Phase-II- und -III-Studien.
\end{abstract}

den die Patienten, die zu irgendeinem Zeitpunkt abbrachen $(\mathrm{n}=176)$, mit den Nichtabbrechern $(n=231)$ verglichen.

Nach einem Follow-up von mindestens 18 Monaten war das mittlere progressionsfreie Überleben in der Gruppe, die die Therapie fortsetzte, nicht signifikant länger als unter den Therapieabbrechern (10,8 vs. 8,4 Monate; $p=0,97)$. Der Median für das Gesamtüberleben war in beiden Gruppen noch nicht erreicht $(\mathrm{p}=0,23)$. Die objektive Ansprechrate war unter den Abbrechern in der Induktionsphase sogar etwas höher als unter den übrigen Patienten (58,3 vs. 50,2\%). Die meisten schweren Nebenwirkungen traten während der Induktionsphase auf; sie ließen sich in der Regel gut behandeln.

Christian Behrend

Schadendorf D et al. Efficacy and Safety Outcomes in Patients With Advanced Melanoma Who Discontinued Treatment With Nivolumab and Ipilimumab Because of Adverse Events: A Pooled Analysis of Randomized Phase II and III Trials. J Clin Onc. 2017; https://doi.org/10.1200/ JCO.2017.73.2289.

\section{Kombinierte BRAF-/MEK-Inhibition bei BRAF- V600-mutiertem Melanom: Langfristig vorteilhaft}

\begin{abstract}
Die Kombination aus dem BRAF-Inhibitor Dabrafenib und dem MEK-Inhibitor Trametinib schneidet bei Patienten mit fortgeschrittenem BRAF-mutiertem Melanom auch in der Langzeitanalyse besser ab als die Monotherapie.
\end{abstract}

$\int$ chon die ersten Analysen der COM$\int$ BI-d-Studie hatten gezeigt, dass die kombinierte BRAF-/MEK-Blockade mit Dabrafenib und Trametinib bei Patienten mit BRAF-V600E/K-mutiertem metastasiertem malignem Melanom das progressionsfreie Überleben (PFS) und Gesamtüberleben (OS) verbessern kann. Nun wurden die Daten nach 36-monatigem
Follow-up präsentiert. In der Phase-IIIStudie waren 423 Patienten mit zuvor unbehandeltem inoperablem BRAF-mutiertem Melanom im Stadium IIIC oder IV randomisiert mit Dabrafenib (150 mg 2-mal täglich) plus Trametinib (2 mg 1-mal täglich) $(\mathrm{D}+\mathrm{T} ; \mathrm{n}=211)$ oder Dabrafenib plus Placebo $(D+P ; n=212)$ behandelt worden.

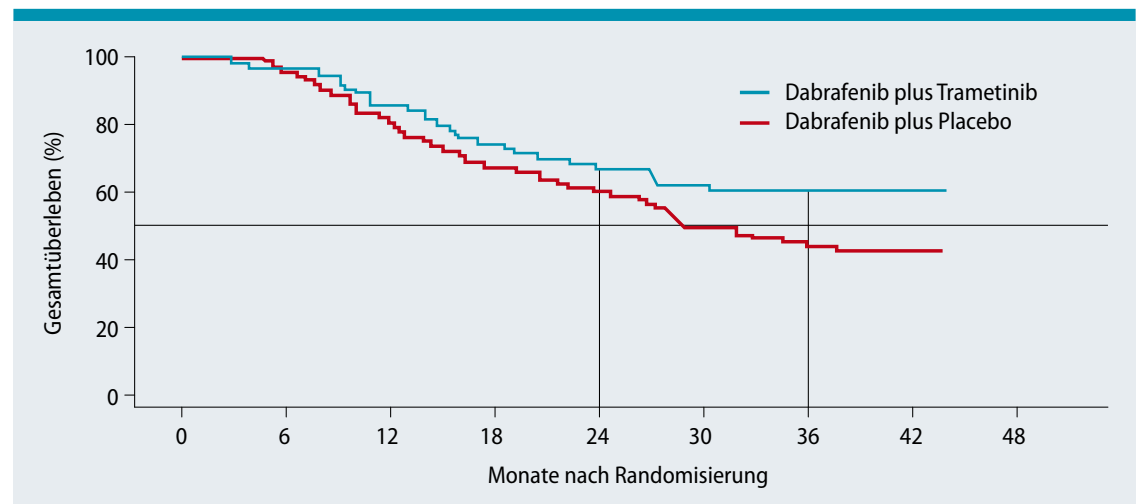

Abb. 1: Patienten mit normaler LDH und weniger als 3 von Metastasen befallenen Organen profitieren am deutlichsten von der Kombination Dabrafenib plus Trametinib.
Die Rate für das 3-Jahres-PFS betrug unter D + T $22 \%$, unter D + P $12 \%$ (Hazard Ratio [HR] 0,71; 95\%-Konfidenzintervall [KI] 0,57-0,88), die Rate für das 3-Jahres-OS lag bei 44 bzw. $32 \%$ (HR 0,75; 95\%-KI 0,58-0,96). 25 (12\%) Patienten, die zunächst mit der Monotherapie behandelt worden waren, wechselten auf die Kombinationstherapie, 6 (24\%) von ihnen hatten zuvor unter der Monotherapie einen Progress erlebt. Von den 76 Patienten der Kombinationsgruppe, die noch am Leben waren (davon 31 progressionsfrei), führten nach 3 Jahren $58 \%$ die Therapie mit Dabrafenib und Trametinib noch immer weiter.

In der Gruppe mit den günstigsten Voraussetzungen (normale $\mathrm{LDH}$ und Metastasen in $<3$ Organen) lag die 3-JahresOS-Rate bei $62 \%$ (Abb. 1), in der Gruppe mit ungünstigen Voraussetzungen (erhöhte LDH) waren es nur $25 \%$. Neue unerwartete Nebenwirkungen traten auch unter der Langzeitanwendung nicht auf.

Fazit: Diese Ergebnisse unterstützen das Konzept einer Langzeittherapie mit Dabrafenib und Trametinib bei Patienten mit fortgeschrittenem BRAFV600-mutiertem Melanom. Kathrin von Kieseritzky

Long GV et al. Dabrafenib plus trametinib versus dabrafenib monotherapy in patients with metastatic BRAF V600E/K-mutant melanoma: long-term survival and safety analysis of a phase 3 study. Ann Oncol. 2017;28(7):1631-9. 